\title{
Lo arcaico y lo moderno en La utopía arcaica de Mario Vargas Llosa
}

\author{
SANTiago López Maguiña \\ Universidad Nacional Mayor de San Marcos \\ slopezgoro@gmail.com \\ $\&$
}

\section{Resumen}

En este artículo se demuestra que el contraste entre lo arcaico y lo moderno en la cultura andina no es presentado por Vargas Llosa solo como una oposición entre dos tipos de racionalidad, la mítica vs la científica, sino entre dos modos de aproximación a la realidad disimétricos, que no poseen términos de comparación y que, por tanto, serían tanto ininteligibles entre sí, como incompatibles. Pero la incomprensión es más acusada desde el lado indígena, que desde el lado criollo, integrado a la vida moderna y a la cultura occidental. Desde la orilla de lo moderno se puede acceder a la captación de lo arcaico, mientras que desde la banda andina es imposible alcanzar el otro borde.

Palabras claves: Peruano, Indio, Mítico, Científico, Arcaico, Moderno, Literatura indigenista.

\begin{abstract}
This article shows that the contrast between the archaic and the modern in the Andean culture is not presented by Vargas Llosa only as an opposition between two types of rationality, the mythical vs scientific but between two ways of approaching dissymmetric to the reality, that have no terms of comparison and thus would be both mutually unintelligible, as incompatible. But the misunderstanding is more pronounced from the Indian side, since the criollo hand, integrated into modern
\end{abstract}


SANTiago López Maguiña

life and Western culture. From the edge of the modern you can access the uptake of the archaic, while from the Andean band is impossible to reach the other edge.

Key words: Peruvian, Indian, Mythical, Scientist, Archaic, Modern, Indian Literature.

Mario Vargas Llosa en La utopía arcaica (1996) escribe: “'lo peruano' no existe. Solo existen los peruanos, abanico de razas, culturas, lenguas, niveles de vida, usos y costumbres, más distintos que parecidos entre sí, cuyo denominador común se reduce, en la mayoría de los casos, a vivir en un mismo territorio y sometidos a una misma autoridad" (210). El premio nobel comenta una afirmación de Jorge Basadre, quien sostenía que la "personalidad" peruana era una "conjugación" de dos factores: la cultura aborigen, "sumergida por la conquista española" (210, Basadre citado por Vargas Llosa) y la "expansión europea de la que somos, en realidad, solo uno de sus muchos exponentes" (Idem).

Para Vargas Llosa no existe tal conjugación. Lo "peruano" es una cualidad expresiva sin contenido: un signo y un enunciado sin significado. En cambio, los "peruanos" si poseen componentes definitivos procedentes de la circunscripción espacial significativa, denominada Perú en la cual pueden ser identificados y reconocidos. Los "peruanos" definidos por su pertenencia a un territorio no forman, sin embargo, un conjunto homogéneo. Son individuos cuyo único común denominador es compartir un mismo espacio. Se diría en ese sentido que son vecinos, antes que integrantes de una comunidad donde todos participan del sentimiento de formar parte de una misma colectividad familiar.

Pero el territorio para Vargas Llosa no es una condición determinante para la constitución de una colectividad nacional, como si lo es para importantes autores peruanos como Luis E. Valcárcel, Luis Alberto Sánchez y José Carlos Mariátegui, quienes escriben sobre el tema en las décadas del 20 y del 30 del siglo pasado, y asumen que el medio ecológico y geográfico es condición necesaria en la definición de lo peruano. Vargas Llosa piensa que el territorio no da un sello de identidad. En todo caso es una condición circunstancial irrelevante en la constitución de una colectividad nacional. Aunque sí lo es en cuanto se establece en él un Estado, el cual circunscribe un espacio en el que impone una regulación de manera artificial. En la definición de lo "peruano" no se encuentra, por tanto, un factor natural que de por sí transmita a un grupo de individuos el sentimiento de pertenecer a una misma entidad colectiva. Existe, 
en cambio, un factor artificial como el Estado, una excrecencia diría Badiou, que obliga a los hombres que ocupan un territorio a asumir que son parte de una comunidad.

Lo nacional, entendido como una cualidad definitoria de una identidad colectiva, se reconoce en cambio por una serie de rasgos constitutivos, entre los cuales no se encuentra el territorio. Ellos son la raza, la cultura, la lengua, el nivel de vida, los usos y costumbres, que en el Perú son múltiples y disimiles. Mario Vargas Llosa subraya, sin embargo, la disimetría que caracteriza a los peruanoa, la diferencia radical que se da entre ella, manifiesta en tres niveles: en el tipo de "civilización", en el grado de comunicación entre los habitantes de ese país y, por último, en el grado de aceptación de una autoridad estatal de alcance nacional.

El grado de dispersión y de separación de los peruanos entre sí es inmenso, desde el punto de vista de los tipos de "civilización" en que viven los diferentes grupos humanos: "Hay peruanos que no han salido de la Edad de Piedra, y otros que están ya en el siglo XXI” (Ibid.). Unos viven aún de la caza y de la recolección, no han llegado a dominar la agricultura, la ganadería ni los metales, mientras otros interactúan con máquinas y equipos sofisticados pertenecientes al mundo del futuro. La distancia técnica y cognoscitiva que aleja a aquellos de los que viven en la era de la ciencia y de la información no es sólo gradual. Es una distancia radical, referida a formas de vida que no pueden interrelacionarse. Mario Vargas Llosa, quien sigue a Karl Popper, destaca que esas formas de vida se definen por los modos (o estrategias) con que los hombres se relacionan con la realidad. Los primeros lo hacen de un modo mágico-religioso, en tanto los segundos de un modo técnico científico.

Unos se hallan bajo el dominio de la creencia, los otros influidos por el saber. Aquellos asumen que la naturaleza está formada por seres y fuerzas invencibles, los cuales imponen su voluntad en forma irremediable; los segundos pueden explicar los movimientos del mundo natural al punto de controlarlos y modificarlos, no siempre de manera inmediata, por cierto, pero de todas maneras en perspectiva de lograrlo en algún momento.

El modo de relación mágico-religioso con el mundo es representado por Mario Vargas Llosa a partir de las experiencias que describe José María Arguedas en Los ríos profundos. El contacto con la naturaleza que el niño Ernesto experimenta muestra "en germen una concepción animista del mundo" (185), propia de la visión de la población indígena. Según ésta plantas, animales y objetos aparecen como seres dotados de ánima, esto es, de capacidad de razonamiento y de voluntad, con los cuales los hombres pueden interactuar, esto es, 
pueden comunicarse y participar en prácticas comunes. Siguiendo la descripción que hace Vargas Llosa de la actuación de Ernesto con respecto al mundo natural y el mundo de los objetos, que incluye objetos fabricados y modernos, se puede precisar las características de "la concepción animista del mundo".

El "entusiasmo por la naturaleza" vivido por Ernesto, dice Vargas Llosa, es "de raíz compensatoria” y "colinda con el embeleso místico" (185). Ello se puede apreciar en el estado de extrema sensibilidad y reducida capacidad intelectiva en la secuencia sobre el "espectáculo de la aparición del sol en medio de las lluvias dispares [que] deja al niño 'indeciso' y anula en él la facultad de razonar" (Ibid.). Ese "arrobo - afirma el escritor -, contiene en sí una verdadera alienación y entraña en germen una concepción animista del mundo" (Ibid.). Refiere a continuación que la "sensibilidad, exacerbada hasta el ensimismamiento por la realidad natural, lleva a Ernesto a idealizar paganamente plantas, objetos y animales, y a atribuirles propiedades no solo humanas, también divinas: a sacralizarlas" (Ibid.) Por fin, concluye: "Desde su condición particular, Ernesto reproduce un proceso que el indio ha cumplido colectivamente y es por ello un personaje simbólico" (Ibid.)

El proceso de asunción de las creencias mágico-religiosas que vive el "indio", ejemplarizado por las experiencias sensitivas de Ernesto respecto de la naturaleza, corresponde al recorrido de un acontecimiento que comienza en un "entusiasmo", sigue con un "embeleso" y un "arrobo", y termina en subyugación. Ese recorrido puede ser captado bajo los esquemas de la semiótica tensiva y sería interesante hacerlo, pero acá sólo se van a destacar algunos rasgos significativos de un análisis preliminar. El "entusiasmo", exaltación del ánimo ante la aparición de algo imprevisto o magnífico (que surge ante la mirada asombrada por algo inesperado) es un estado en el cual la subjetividad, entendida como la capacidad o la competencia de actuar, es trastornada, aún más, pasmada; desde el punto de vista figurativo, petrificada (Zilberberg: 2007: 179 y ss). Se trata de la experiencia de un detenimiento vivencial, de un embobamiento, como resultado de un sobrecogimiento desmesurado. De allí que el hombre no sólo quede cautivado, enlazado a una presencia arrebatadora, sino sobre todo preparado para ser invadido. Esta penetración de lo otro, que siempre es extraño e inexplicable, presupone una subyugación a algo que se percibe superior, y que en la fase mágica de la existencia humana es captado tanto como provisto de voluntad, como de poderes cuya fuerza es de una dimensión que sobrepasa a las fuerzas de la cultura.

Dos rasgos derivados del pensamiento mágico, el ensimismamiento y el paganismo, son características que en el discurso criollo se atribuyen al indio. Se los encuentra en la narrativa de Ventura García Calderón, el más importante 
autor indianista, según la clasificación realizada por Tomás Escajadillo. Son indianistas autores que hacen en sus discursos una representación de los indios y de lo indio como presencias de otro mundo extraño y salvaje, en oposición al mundo propio del enunciador, que se ubica en un mundo familiar e ilustrado. En dos conocidos relatos de Ventura García Calderón los indios son configurados como seres silenciosos, encerrados en sí mismos, en un ensimismamiento que procede de su alma sumisa y cobarde, parte de un mundo misterio, inescrutable y lleno de amenazas. Lo mismo, en la narrativa indigenista de Enrique López Albujar, el indígena andino es descrito como un ser absorto en sí, pero taimado, capaz de las peores alevosías, y por quien debe guardarse cuidado, de la misma manera que por las fieras que acechan a sus presas en silencio, aunque el mundo en que vive es próximo y conocido al enunciador.

Lo distintivo del discurso de Mario Vargas Llosa es que el ensimismamiento indio en la contemplación de la naturaleza deja que su cuerpo sea invadido y aprisionado por las entidades que forman parte de aquella. Y se deja atrapar por un embeleso, casi místico, por una fascinación que lo inhabilita para todo razonamiento. Eso sucede en la experiencia de la sorpresa, que ocurre al sujeto ante una aparición súbita, que lo sobrecoge y lo avasalla, estado del que sin embargo es capaz de restablecerse y recomponerse con cierta rapidez (Zilberberg:2007: 179 y ss). El sujeto animista, mágico-religioso que Mario Vargas Llosa encuentra en la novela de José María Arguedas, queda en cambio suspendido en un arrobamiento permanente, petrificado en ese estado. Aquí habría que seguir la configuración de lo pétreo como una de las expresiones más distintivas de la cultura andina, predicadas en los discursos tanto criollos como andino mestizos.

El adjetivo "pagano" para calificar la actuación de Ernesto, el personaje principal de los Ríos profundos, emparienta el discurso de MVLL con la visión de los autores del siglo XVI y del siglo XVII respecto de las creencias y las prácticas religiosas de los nativos en América. Se llamaba paganas a las creencias y prácticas que no eran cristianas, y en especial a aquellas que asumían la existencia tanto de muchos dioses, como del carácter sagrado de cualquier entidad considerada poderosa. El paganismo de un grupo humano era percibido y aún lo es por quienes profesan una religión monoteísta, o por quienes ven en ese rasgo una propiedad superior, como una manifestación de salvajismo e ignorancia, o como en el caso de MVLL una expresión de irracionalidad.

La sensibilización extrema que desata la contemplación del mundo natural es producto de una situación en particular. El personaje de la citada novela de Arguedas, además de haber crecido dentro de la cultura indígena, cuidado y 
educado por los indios, está ligado a ella "subconscientemente" (sic) (185) por su "propia situación" (Ibid.) de niño "exilado", que no encuentra arraigo entre los hombres de su clase. Ernesto ha sido matriculado como alumno interno en Abancay, capital del departamento de Ayacucho, donde vive por identificación duras experiencias de ajustamiento en un mundo de jerarquías, violencia y exclusión de los más débiles y los indios, a pesar que él es blanco. Esa situación para MVLL permite a Ernesto reproducir "un proceso que el indio ha cumplido colectivamente y es por ello un personaje simbólico" (ibid.). De ese modo, "como para el comunero explotado y humillado en todos los instantes de su vida, sin defensas contra la enfermedad y la miseria, la realidad difícilmente puede ser lógica, para el niño paria, sin arraigo entre los hombres, exilado para siempre, el mundo no es racional sino esencialmente absurdo" (Ibid.) Esa circunstancia explica "su irracionalismo fatalista, su animismo y ese solapado fetichismo que lo lleva a adorar con unción religiosa los objetos más diversos" (Ibid.).

Para MVLL en conclusión las creencias y las prácticas mágico-religiosas nacen de una imposición de la naturaleza sobre el hombre. De su aparición muy intensa y concentrada, que lo cierra, lo impacta y lo convierte en un individuo deslumbrado y pasmado hasta el retraimiento. Pero este estado no se produce de un contacto sólo sensible (sólo estésico o tímico se diría en términos de la semiótica greimasiana (Greimas: 1989), sino también de un estado de cosas. Quienes se embriagan y enajenan con la naturaleza son aquellos que han sufrido o sufren explotación y humillación de amos despiadados que los deja en el desamparo, que padecen de "la enfermedad y el hambre", experiencias inexplicables y absurdas. En todo caso, en términos más generales, hay una grave situación de falta en el inicio de la cadena de estados y transformaciones que hace de los sujetos actores predispuestos a sucumbir a la fascinación del mundo natural. Es una situación de debilidad que los inclina hacia la sumisión a entidades poderosas, a las cuales no pueden enfrentar. Todo lo cual es percibido como algo absurdo.

Hay, por consiguiente, un estado de flaqueza inicial expresada bajo la forma de una sensibilidad excesiva (muy intensa y concentrada) y una racionalidad exigua (de poca intensidad y difusa). Este estado hace de los sujetos que la padecen propensos al sobrecogimiento, al arrobamiento y subyugación que les producen presencias poderosas y admirables. Este es el esquema por tanto que explica las creencias y las prácticas mágico religiosas: ellas presentan un carácter estésico, son fruto de una estimulación o tonificación corporal fluida, que no ofrece trabas; son fruto del dominio de lo sensible, componente primario de la corporalidad y de la significación (Greimas:1990 y Fontanille: ). En oposición, se deduce, que los conocimientos científicos tienen un carácter 
estético: son fruto de una dinamización de la inteligibilidad, componente segundo de la corporalidad y de la significación, la cual, en cambio, ofrece dificultades para la realización de la sorpresa. Se observa entonces que la sensibilidad es dimensión perceptiva que se activa cuando el mundo natural puede impresionar sin resistencias al observador. La inteligibilidad, en cambio, es dimensión perceptiva que ofrece resistencias. Gracias a la inteligibilidad el sujeto tantea, mide, calcula, toma distancia respecto de las presencias que le conmueven. Es sujeto que no se deja invadir de inmediato, en el que predomina la inteligibilidad sobre la sensibilidad y es capaz de operar tanto sobre el mundo exterior e interior. Es capaz de modificarlos. El sujeto, en cambio, en el que es más importante la sensibilidad se deja simplemente someter.

Pero hay un punto que parece contradecir este esquema. La subyugación que ejerce el mundo natural sobre el indio es producto también de la percepción de su situación de sumisión y de carencias como una situación absurda, como una situación que carece de sentido y que lo obliga a buscar la compensación de la magia y de la religión. Es de observar que la falta de sentido, que aparece como una manifestación de lo inexplicable, de lo que carece de lógica, no se puede captar sino en la medida en que puede contrastarse con lo que tiene sentido, con lo que es explicable, con lo que tiene lógica. No es seguro, sin embargo, que MVLL defina lo "absurdo" sólo en esa dirección. Lo más probable es que esa expresión se refiera también a la experiencia penosa de un estado de carencia respecto a un ideal de plenitud o de satisfacción esperable, que el sujeto no logra alcanzar y que lo vive como una experiencia ingrata que juzga injusta, es decir, no proporcional, y que tampoco puede modificar. Este estado, de vivencia de una imposibilidad, suscita a continuación en quien la padece una voluntad compensatoria, que encuentra en las creencias y prácticas mágico-religiosas el medio de conseguirla. Ellas le permiten sobrellevar sus sufrimientos, mediante la realización imaginaria de deseos imposibles.

Se podría sostener finalmente que las creencias mágico-religiosas nacen de una imperfección que tanto afecta el estado de cosas (la carencia en general, la carencia física y material), como el estado de ánimo (una disposición inherente al arrobamiento, aunque alimentada por un estado de falta material, entendido en el contexto de este artículo como la privación de bienes indispensables para la supervivencia).

Para Mario Vargas Llosa, por último, los hombres que conviven bajo una forma de vida mágico-religiosa actúan de acuerdo a estrategias de sobrevivencia, que privilegian las prácticas rituales, basadas en intercambios simbólicos con las divinidades del mundo natural, montañas, tierra, ríos, lagos, manantiales, cascadas, plantas, animales, fenómenos naturales, etc. Esos individuos 
desarrollan tales prácticas como consecuencia de los estados de carencia que padecen, las cuales acentúan una disposición atávica al arrobamiento irracional respecto a presencias que parecen indomeñables, a las que atribuyen voluntad y consciencia. A esas entidades se les otorga dones por los que se espera recibir de ellas en reciprocidad contra-dones, bienes equivalentes o incluso superiores.

Los hombres que conviven bajo una forma de vida científica operan por su parte de acuerdo a estrategias que buscan la comprensión racional del mundo natural y que procuran su control y transformación mediante prácticas de observación, descripción, medición, verificación de hipótesis, ensayos y pruebas. Aquellos han superado el estado primigenio según el cual se cree que la naturaleza está formada por seres sobrenaturales dotados de voluntad y que pueden, en consecuencia, actuar sobre ella para adaptarla a sus necesidades. El hombre en este caso se ve a sí mismo como un personaje más poderoso y capaz de someter a las distintas entidades del mundo natural, sino de manera inmediata, en proyección. Aquel va en camino definitivo de convertirse en dueño completo de la naturaleza.

Ahora bien Vargas Llosa no desarrolla, sin embargo, un esquema tan preciso para el caso de la forma de vida científica como para la forma de vida mágico religiosa. Ya se han adelantado unas pocas observaciones sobre ella, y no son muchas más las que se pueden añadir, pues el novelista no abunda mucho al respecto. Pero hay un punto decisivo: presenta a la cultura científica en oposición a la mágico-religiosa. "Una cultura mágico-religiosa puede ser de un notable refinamiento y de elaboradas asociaciones -de hecho, lo son la mayoría de ellas-, pero será siempre primitiva" (187) respecto de "la cultura moderna" (Ibid.). Vargas Llosa alude aquí al carácter sistemático de la cultura mágico-religiosa, en cuanto a la composición de sus conexiones, de carácter analógico o metafórico. Precisa, sin embargo, que esa sistematicidad no es suficiente para que esa cultura deje de ser primitiva. El constituyente que parece determinar esa condición es su carácter tribal y cerrado. Puede imaginarse que es carácter que afecta a su grado de generalización. La cultura mágico-religiosa lograría a lo más representaciones y sistematizaciones solo válidas para una comunidad, limitadas a ella y de restringidas posibilidades argumentativas y explicativas. La cultura científica, en oposición, es una cultura abierta, capaz de generalizar sus enunciados más allá de lo local además de contar con amplias posibilidades argumentativas y explicativas. Y ello porque está fundada en una racionalidad y una actitud que "subordina el conocimiento a la experiencia y al cotejo de las ideas y de las hipótesis con la realidad objetiva (Ibid.)". Vargas Llosa repite una y otra vez en sus ensayos sobre la obra de José María Arguedas el enunciado de que el conocimiento científico 
se consigue gracias a hipótesis verificadas o falseadas con la realidad objetiva y no se ocupa de hacer más elaboraciones al respecto. Le basta con referir que en ese punto sigue lo planteado por Karl Popper The Open Society and Its Enemies. Le importa, sin embargo, subrayar que el conocimiento científico es un modo de captación del mundo superior al mágico en cuanto permite a los hombres superar "la realidad colectivista de la horda y la tribu" y lograr "la comunidad de individuos libres y soberanos" (Ibid.).

Individuos que participan de formas de vida tan opuestas no pueden comunicarse entre sí. Unos viven según una lógica que propicia prácticas pasivas, que hacen de los seres humanos actores más dispuestos a recibir que ha producir; otros, en cambio, viven según una lógica de prácticas dinámicas, consistente en la adquisición de lo necesario para su existencia y más aún sobrepasar el estado de necesidad mediante el aprovechamiento controlado de la naturaleza, que no se contenta con una satisfacción inmediata y diaria, sino que se anticipa a fin de evitar cualquier situación eventual de carencia, y, mucho más, que apunta a una acumulación de bienes (acumulación de riqueza, gusta decir Vargas Llosa) que anule la previsiones más pesimistas y proporcione satisfacción y seguridad.

Así se encuentran en el territorio llamado Perú grupos sociales a los que les alcanza con sobrevivir, al amparo de sus dioses tutelares o los espíritus benefactores, con otros que persiguen un tipo de vida capaz de asegurarles una existencia sin riesgos ni incertidumbres en la medida en que la naturaleza pueda sea mejor entendida, controlada y transformada. Se trata de dos sistemas por completo disimétricos, que no pueden comunicarse entre sí. Uno asume la escasez y la subyugación de lo más fuertes como una fatalidad, otro mira por la abundancia y su logro se estima posible si se cumple la condición del dominio de lo aparentemente indómito y arisco.

Pero el acuerdo entre los peruanos depende de un factor más primordial aún que el de las diferencias culturales y linguiísticas. Es el referido a los distintos intereses de los grupos sociales y culturales, que se resisten a acatar un orden estatal. La convivencia entre los peruanos no es voluntaria sino impuesta, obligada por una autoridad, por un Estado. Pero esto, dice el novelista, tampoco es del todo cierto, "pues ni siquiera las leyes a que en teoría la sociedad entera está sujeta rigen para todos los ciudadanos de la misma manera, ni los problemas se comparten de modo que podría considerarse semejante, equitativo, o aun aproximado" (Ibidem.). En el nivel jurídico-político la presencia del Estado no logra hacer de los habitantes del territorio peruano ciudadanos con los mismos derechos y deberes, porque no todos se reconocen como parte de él, ni porque tiene los medios ni la voluntad para reconocerlos. Es más, los in- 
dividuos y los grupos no se reúnen alrededor de intereses idénticos, equivalentes o, incluso, más o menos cercanos. La dispersión es muy acentuada y amplia.

Los variados intereses, sin duda, se han gestado en las prácticas y las estrategias propias de las formas de vida que antes se han presentado de manera esquemática. Unos son los intereses que corresponden a la finalidad de sobrevivencia, otros a la finalidad de la abundancia y la seguridad, que no se pueden condecir. Pero además se añaden a ellos otros de muy diversa naturaleza, que en general coinciden en la resistencia a la autoridad, motivadas por un acusado sentimiento de rivalidad contra el diferente o por un resentimiento irrevocable. Estos son puntos que Mario Vargas Llosa no desarrolla, sin embargo, con amplitud y se limita a unas pocas observaciones. Lo hace en otro libro, en las memorias que escribió después de la derrota en las elecciones presidenciales de 1990, en las fue elegido Alberto Fujimori, Como pez en el agua.

Las formas de pensamiento mágico-religiosas son parte de la "cultura india” (29) que según Mario Vargas Llosa "fascinaba” a José María Arguedas, "y que hubiera querido conservar" (Ibid.). La "cultura india" para el escritor es "el sistema de supervivencia que ha permitido al indo de los Andes (...) mantener en el dominio de la lengua, de las costumbres, de los ritos, una cierta continuidad con el pasado" (Ibid.) y, a la vez, "transformar las instituciones, creencias y aun la lengua que fueron impuestas por la cultura occidental o hispana en algo sustancialmente distinto" (Ibid.). Arguedas amaba el "carácter 'arcaico' y 'bárbaro' de la realidad india -lo tradicional y lo metabolizado de la cultura de Occidente-" (pp. 30-31). Ese amor por lo arcaico colisionaba, sin embargo, en su discurso o en su pensamiento "con las fuerzas e ideas progresistas" de José Carlos Mariátegui y del APRA, quienes "consideran que la única manera de salvar al indio es liberándolo, al mismo tiempo que de sus explotadores, de las supersticiones, usos bárbaros y ritos retrógrados" (31).

En la percepción del mundo andino corren en el discurso de Arguedas dos orientaciones de tensiones polares. Una lo apega a creencias y a prácticas supérstites de tipo arcaico, y otra lo aproxima a principios que afirman una visión moderna, fundados en la ciencia. Esa oposición no se presenta, sin embargo, como una alternativa objetivable, como un contraste entre términos elegibles del mismo valor, pues si bien Arguedas tiene una formación científica y moderna, al mismo tiempo siente una "identificación profunda", por las "creencias, ritos, músicas, magias, sacrificios votivos de animales" (163). Mario Vargas Llosa anota a continuación que todas esas manifestaciones "son la supervivencia de un esplendoroso pasado, de un ideal arcádico de total armonía entre la 
sociedad humana, el mundo trascendente de los dioses y la naturaleza sin domeñar" (Ibid.).

La simpatía por la "cultura india" aparece, de acuerdo a la última anotación, como una estimación intensa y circunscrita hacia expresiones que sobreviven a una época ya cancelada o en vías de extinguirse. La predilección por "restos" que no tienen lugar, que no tienen sentido, que no tienen significación en los procesos de cambio de la sociedad moderna. Piezas de museo, muestras de materiales fuera de uso, presencias de un pasado remoto. O que están en camino hacia esa inservible condición. Remiten a la antigua cultura inca o las viejas culturas prehispánicas, que de acuerdo al imaginario indigenista y de los propios nativos peruanos vivieron en asombrosa armonía con la naturaleza y con los dioses tutelares, culturas que el hombre andino de hoy añora y que quisiera restablecer, como se expresa en el mito de Inkarrí, relato al que Arguedas hizo referencia muchas veces desde que fue recogido en la comunidad Q'ero por Oscar Núñez del Prado, antropólogo de la Universidad San Antonio Abad de Cusco. Inkarrí es una divinidad fragmentada por los españoles, cuyas partes diseminadas por distintas zonas del Perú se hallan reconstituyéndose, y se espera que llegará el día en que esa recomposición termine. En ese momento el mundo del pasado se restablecerá. Este relato en los términos y en la lógica del mito coincide con el discurso indigenista y del propio Arguedas acerca de un pasado armónico y esplendoroso, al que la cultura andina debería y aspira volver, y que Vargas Llosa denomina "utopía arcaica" (163). El mito de Inkarri, por eso es calificado por el Premio Nobel como "un emblema de resurrección de aquella utopía" (Ibid.).

La "utopía arcaica" es, por tanto, un ideal de vida según el cual el hombre del ande cree que sus antepasados vivieron en otro tiempo, antes de la colonización española, en armonía con la naturaleza percibida como una totalidad formada por entidades humanizadas con las cuales el hombre interactúa. Ideal que, a pesar de los tiempos modernos que se viven en el mundo, aun sobrevive en la población indígena de los Andes.

En el imaginario de José María Arguedas, como en el de los indios, la opción entre la "utopía arcaica" y la vida moderna se inclina hacia la primera de una manera instintiva, "aun cuando la modernización -dice Vargas Llosa- de la región andina era inevitable e indispensable" (Ibid.). Escribe Vargas Llosa que en el "El ayla" "se advierte esa escisión" (Ibid.). En ese relato "Arguedas parece aprobar racionalmente el proceso de mestizaje" que observa "en el pueblo de su infancia y que los propios indios quieren acelerar pues la saben herramienta eficaz para resistir la injustica" (Ibid.). Ocurre, sin embargo, "oscuramente, se percibe a lo largo del texto una profunda melancolía por la profunda melancolía por la 
desaparición de la sociedad tradicional y una suerte de instintivo horror ante la perspectiva de aquella comunidad donde imperaba el colectivismo solidario y mágico de antaño vaya rumbo hacia “el individualismo escéptico”' (Ibid.).

El antagonismo entre lo arcaico y lo moderno convive con su concierto o su conciliación, pero tan pronto el segundo término de la oposición empieza a imponerse, surgen fuerzas instintivas en favor de la resistencia y el reforzamiento del primer término, y el antagonismo y la escisión sientan su dominio. La superioridad de lo arcaico, sin embargo, no es posición de larga duración, porque de manera ineluctable la modernización va a establecer un mundo de hombres libres tanto de las ilusiones supersticiosas y paganas, como de las injusticias que seres humanos infringen a otros. De hecho la suposición de un retorno al pasado es imposible, lo que hace de cualquier pretensión en ese sentido una aspiración absurda, incluso un signo de perturbación.

El contraste entre lo arcaico y lo moderno en la cultura andina no es presentado por Vargas Llosa como una oposición entre dos tipos de racionalidad, la mítica vs la científica, sino entre dos modos de aproximación a la realidad disimétricos, que no poseen términos de comparación y que, por tanto, serían tanto ininteligibles entre sí, como incompatibles. Es de suponer, sin embargo, que esa incomprensión es más acusada desde el lado indígena, que desde el lado criollo, integrado a la vida moderna y a la cultura occidental. Desde la orilla de lo moderno se puede acceder a la captación de lo arcaico, mientras que desde esta banda es imposible alcanzar el otro borde. El hombre obnubilado y embelesado por el mundo natural, no podría ni aproximarse a una aprehensión distanciada y objetiva de ese mundo. Esa visión no entraña, sin embargo, la hipótesis de una incapacidad innata en el hombre andino para acceder a los conocimientos y al pensamiento moderno. Todo lo contrario, él se ve impulsado a acercarse a estos a fin de superar el estado de explotación y opresión que lo sume en la miseria. Ello quiere decir que tiene una aptitud potencial, a veces actualizada, pero no aún realizada para asimilar las formas del pensar y del vivir moderno.

De esa formulación se deduce que habría un determinante externo que operaría como obstáculo para conseguir esa transición. Cierto, Vargas Llosa ve que las ideas progresistas de izquierda son las que originan contraprogramas narrativos respecto del programa de la modernización. Pero también hay factores internos: impulsos y pulsiones oscuras que pugnan por la conservación de un hombre andino inmerso en un mundo dominado por el asombro y el sortilegio de presencias que parecen proveer y despojar a voluntad los bienes necesarios para la sobrevivencia. Miedo y amor por seres que no se entienden de otra manera que como proyección imaginaria de la propia subjetividad. 
Los dos factores que impiden la transición de lo arcaico a lo moderno son oscuros y enrevesados, al mismo tiempo que nacidos de una perturbación, de algún tipo de trauma que trata de ser superado mediante el delirio de un orden que pudiera conjugar bienestar con colectivismo. Delirio del retorno a un pasado armonioso, de convivencia solidaria entre todos los hombres y de perfecta participación comunicativa con la naturaleza sacralizada. Delirio también, por otra parte, de un futuro de igualdad y de cooperación entre los seres humanos, por fin amos de sí mismos y de la materia. Dos delirios nacidos del agravio y del resentimiento, que se imponen sobre la razón, sobre el criterio sensato y lógico de que el bienestar solo llega con la iniciativa privada, con la imaginación y la creatividad de individuos libres. En el primer caso se adivina el rencor de un sujeto arrancado de un cuerpo al que se halla apegado. En el segundo se percibe el encono de un actor que se siente agredido por el desprecio hacia su persona y por la opresión propia y ajena que sufre de parte de amos soberbios, arbitrarios y crueles.

Según Vargas Llosa no hay modo de amoldar lo moderno con lo arcaico. Ambos son estados mutuamente excluyentes y disimétricos. Para el novelista Arguedas habría deseado que su ajuste fuera posible, pero nunca encontró la forma que eso pudiera ocurrir, puesto que lo moderno se impone de manera inevitable mediante la negación de lo arcaico y este estado mediante la negación de lo moderno. La negación de lo moderno es el progreso que constituye el recorrido por el cual el hombre se separa de la naturaleza y va asumiendo los valores de "la sociedad industrial y de la cultura urbana", así como "de la civilización basada en el mercado" (39). La negación de lo moderno equivale al retroceso o al atraso, que consiste en el rechazo de la industria, de lo urbano, del mercado y de la ciencia. Vargas Llosa, sin embargo, insiste en destacar que el rasgo distintivo de lo arcaico es la superstición y la magia, sustentadas en lo instintivo e irracional, mientras que el rasgo distintivo de lo moderno es la ciencia, sustentada antes que en lo reflexivo, en la demostración de que sus enunciados son falsables, esto es, susceptibles de ser puestos prueba para comprobar que no son falsos y que, por tanto, pueden ser verdaderos, lo cual es una expresión de racionalidad. En el siguiente cuadrado semiótico destacamos los principales elementos significativos del discurso de Vargas Llosa sobre la oposición entre lo moderno y lo arcaico, que resumen el análisis hasta aquí realizado: 


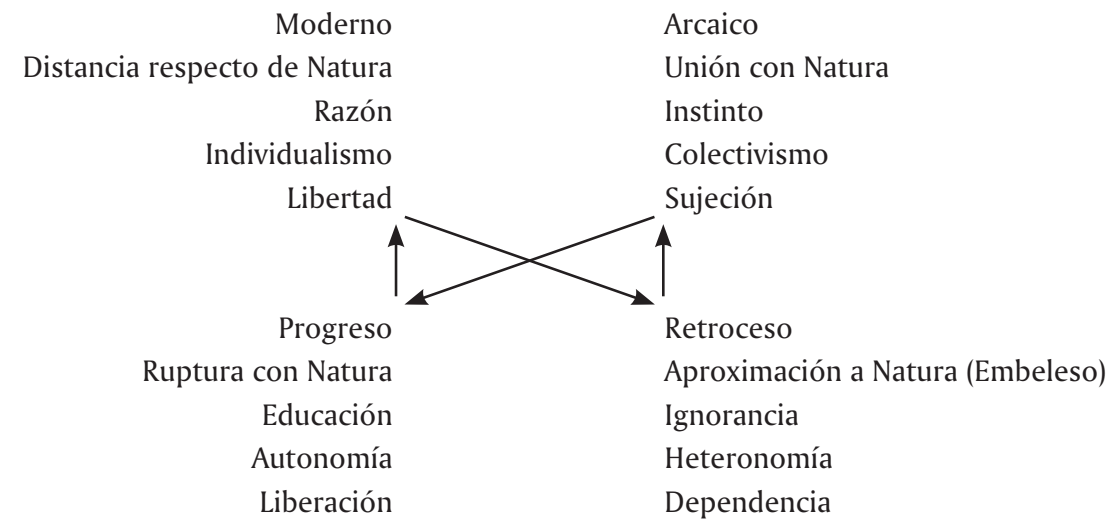

En esta estructura elemental se introduce la categoría de la educación como término que niega lo instintivo, que a su vez se opone a la racionalidad científica. No hay explícitas formulaciones en La utopía arcaica acerca de esa categoría, pero puede ser inferida de varios enunciados. Se puede decir por eso que la educación es el medio o proceso por el cual un individuo puede liberarse de las creencias mágicas y las supersticiones que lo mantienen inmovilizado frente a la naturaleza. Juega un papel central en la vida de Arguedas: ella le permite acceder a la ciencia y a la modernidad. Al lado de la educación también podría ponerse al buen criterio, al sentido común, al que el hombre accede con la práctica del trabajo y la producción de riqueza para su propio bienestar.

\section{Referencias bibliográficas}

FONTANILlE, Jacques (2008) Soma y Sema. Figuras Semióticas del Cuerpo. Lima: Fondo Editorial de la Universidad de Lima.

GREIMAS, A.J. (1990) De la Imperfección. México: Fondo de Cultura Económica.

VARGAS LlOSA, Mario (1996) La Utopía Arcaica. José María Arguedas y las Ficciones del Indigenismo. Lima: Fondo de Cultura Económica.

ZILBERBERG, Claude (2007) Semiótica Tensiva. Lima: Fondo Editorial de la Universidad de Lima. 